\begin{tabular}{|c|l|}
\hline Title & Nanoscale thermoelastic probing of megahertz thermal diffusion \\
\hline Author(s) & Tomoda, Motonobu; Wright, Oliver B.; Li V oti, Roberto \\
\hline Citation & $\begin{array}{l}\text { A pplied Physics Letters, 91/7), 071911 } \\
\text { https://doi.org/10.1063/2770769 }\end{array}$ \\
\hline Issue Date & 2007-08-13 \\
\hline Doc URL & http://hdl.handle.net/2115/28028 \\
\hline Rights & Copyright $\odot$ 2007 A merican Institute of Physics \\
\hline Type & article \\
\hline File Information & APL91-071911.pdf \\
\hline
\end{tabular}

Instructions for use 


\title{
Nanoscale thermoelastic probing of megahertz thermal diffusion
}

\author{
Motonobu Tomoda ${ }^{\text {a) }}$ and Oliver B. Wright \\ Department of Applied Physics, Graduate School of Engineering, Hokkaido University, Sapporo, \\ Hokkaido 060-8628, Japan \\ Roberto Li Voti \\ Dipartimento di Energetica, Università degli Studi di Roma "La Sapienza," Via A. Scarpa 16, \\ 00161 Roma, Italy
}

(Received 21 June 2007; accepted 20 July 2007; published online 14 August 2007)

\begin{abstract}
The authors demonstrate a method to probe thermal diffusion at megahertz frequencies with nanometer lateral resolution in a thin opaque film on a transparent substrate. They map photothermally induced megahertz surface vibrations in an atomic force microscope using tightly focused optical illumination from the substrate side. By comparison with a theoretical model of the surface displacement field, the authors derive the thermal diffusivity of a thin chromium film on a silica substrate. (C) 2007 American Institute of Physics. [DOI: 10.1063/1.2770769]
\end{abstract}

With the continued trend for miniaturization of integrated circuit devices, there has been much interest in the thermal probing of thin films, multilayers, and microstructures down to nanometer length scales. The thermal properties of thin films are, in general, very different from the bulk and depend on defects, grain size, texture, and morphology. ${ }^{1-3}$ Transient or sinusoidally modulated temperature fields are particularly useful for probing thermal properties because the associated thermal wave propagation can be accurately controlled or localized by choice of the time scale. For a modulation frequency $f$ the thermal diffusion length decreases with increasing frequency as $1 / f^{1 / 2}$, taking a value for metals that is typically $\sim 1 \mu \mathrm{m}$ for $f \approx 1 \mathrm{MHz}$. Therefore, it is advantageous when probing thin films with thermal waves to work at high frequencies in order to restrict the measurement volume as much as possible to the film itself.

A particularly versatile noncontact method to transiently heat opaque thin films is to use pulsed or modulated optical beams, either focused to a single spot or crossed to produce induced gratings; time scales from seconds to picoseconds can be accessed in this way. ${ }^{4-8}$ Likewise, optical detection of temperature fields by thermoreflectance or the mirage effect, or of surface displacement fields by optical beam deflection, interferometry, or diffraction have given rise to performant techniques for the noncontact probing of the thermal diffusivity in thin films. ${ }^{8-13}$

However, the lateral resolution of conventional optical detection methods is limited to micron scales owing to the optical diffraction limit. To overcome this limit for purposes of imaging transient thermal fields, the most developed thermal sensing schemes with submicron lateral resolution are based on scanned local probe techniques. ${ }^{14,15}$ Use of a modulated heat source in an atomic force microscope (AFM) tip, for example, has proved useful for imaging time-varying temperature distributions up to frequencies of $\sim 100 \mathrm{kHz}{ }^{15-17}$ AFM-based probing of kilohertz surface vibrations induced by resistive heating or chopped light allows additional contrast from the thermal expansion coefficient. ${ }^{14,18}$

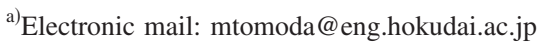

Higher modulation frequencies in the megahertz range-more appropriate for probing thin films of thickness in the $\sim 100 \mathrm{~nm}$ range or below-can be combined with nanometer spatial resolution using a method known as optical heterodyne force microscopy (OHFM). ${ }^{19}$ This detects photothermally induced surface vibrations in an AFM, and can image subsurface nanoscale features through their effect on the surface displacement produced by a megahertz thermal field that is optically excited directly below the scanning AFM tip. To produce a quantitative measurement of the thermal diffusivity in thin films using the combination of megahertz thermal waves and local probing, however, one needs to move the scanning tip laterally with respect to the modulated thermal source in a way analogous to the conventional all-optical methods. ${ }^{6,9,10}$

In the present letter, we present a method based on OHFM to achieve this, involving optical excitation from the transparent substrate side of the sample and AFM detection of surface vibrations from the film side. We demonstrate the method using a thin $\mathrm{Cr}$ film on silica using variable frequency probing in the range of $100 \mathrm{kHz}-4 \mathrm{MHz}$.

Figure 1 shows the modified OHFM setup. We use a commercial AFM system (TM microscopes, CP-M) based on an optical-lever detection system for the bending of a V-shaped cantilever (Ultralever D, length of $85 \mu \mathrm{m}$, width of $28 \mu \mathrm{m}$, thickness of $0.8 \mu \mathrm{m}$, spring constant of $1.6 \mathrm{Nm}^{-1}$,

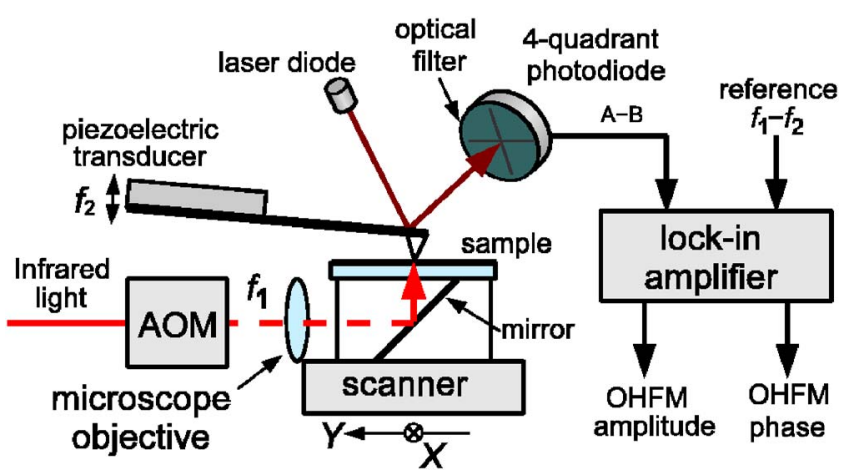

FIG. 1. (Color online) Schematic diagram of the modified optical heterodyne force microscope setup. AOM: acousto-optic modulator. The cantilever is slanted at an angle of $15^{\circ}$ to the sample surface. 
resonant frequency of $170 \mathrm{kHz}$, and tip radius of $10 \mathrm{~nm}$ ). The sample consists of a polycrystalline $\mathrm{Cr}$ thin film with a thickness of $260 \mathrm{~nm}$ made by electron beam deposition on a fused silica substrate. The thickness was independently determined by laser picosecond acoustics. ${ }^{20}$ An $830 \mathrm{~nm}$ laser beam chopped at a frequency $f_{1} \sim 1 \mathrm{MHz}$ is focused on the substrate side of the $\mathrm{Cr}$ film through a $50 \times$ microscope objective lens, producing surface vibrations of small amplitude $\Delta y$ (in the nanometer range) and phase $\phi$ through thermal expansion. The cantilever base is mounted on a piezoelectric slab that is excited at a slightly different frequency $f_{2}$ with an amplitude of $\sim 1 \mathrm{~nm}$. Because of the nonlinear nature of the tip-sample force-distance curve, a vibration of the contacting tip is induced at the difference frequency $f_{1}-f_{2}=3 \mathrm{kHz}$, chosen to be much lower than the fundamental cantilever resonance $(\sim 480 \mathrm{kHz}$ in contact) but much higher than the response frequency of the AFM feedback loop (maintaining a set load of $30 \mathrm{nN}$ ). The amplitude (typically $20 \mathrm{pm}$ here) and phase of the difference-frequency tip vibration is detected by a lock-in amplifier (with time constant $30 \mathrm{~ms}$ ); as explained in more detail elsewhere, ${ }^{19}$ the amplitude is proportional to $\Delta y$, whereas the phase should coincide with the phase $\phi$ of the surface displacement-apart from an additive constant that depends on the elastic properties of both tip and sample. For a sample with a homogeneous surface, one can therefore accurately probe the variations $\Delta y$ and $\phi$ at $f_{1}$ through tip vibrations at the frequency $f_{1}-f_{2}$ while maintaining a constant average distance between the tip and the sample.

The sample is mounted on a stage containing a mirror. This assembly, fixed to the AFM scanner, moves with respect to the tip. When the scanner moves in the $Y$ direction parallel to the axis of the objective lens (see Fig. 1), the optical spot moves together with the sample. (In contrast, the spot moves across the sample surface when the scanner moves in the $X$ direction perpendicular to this lens axis.) The amplitude and phase lag for scans along the $Y$ axis across the center of the optical spot are recorded. The focus of the optical spot, with a full width at half maximum (FWHM) intensity $W=1 \mu \mathrm{m}$, is set when the tip is positioned at the center of the optical spot. $^{21}$

Figure 2 shows $Y$-directed spatial scans of (a) the amplitude and (b) the phase lag of the difference-frequency signal (dots) for the optical chopping frequency $f_{1}=500 \mathrm{kHz}$ for an incident optical power $P=1 \mathrm{~mW},{ }^{22}$ whereas (c) and (d) show the corresponding results for $f_{1}=2 \mathrm{MHz}$ and $P=2 \mathrm{~mW}$, all data being obtained with a $2 \mu \mathrm{m} \mathrm{s}^{-1}$ scanning speed. (We estimate that $P=1 \mathrm{~mW}$, equivalent to $0.6 \mathrm{~mW}$ absorbed power, results in a steady state temperature rise of $80 \mathrm{~K}$ at the $\mathrm{Cr}-\mathrm{SiO}_{2}$ interface, and an oscillating temperature change there at an amplitude of $40 \mathrm{~K}$ combined with a surface vibration amplitude of $0.12 \mathrm{~nm}$ at $f_{1}=500 \mathrm{kHz}$.) The data are obtained by averaging the $+Y$ scans of $128 \times 128$ pixel raster-scanned images.

Immediately obvious from the data is a decrease in amplitude (becoming more pronounced at higher $f_{1}$ ) and an initial increase in phase lag as the tip position $r$ deviates from the spot center $(r=0)$. For larger $r$ the amplitude reaches a constant background governed by the noise level. The slight left-right asymmetry in the graphs is probably caused by residual heat conduction from the heated area through the air to the cantilever that is positioned to the left in Fig. 1.

To elucidate the detected signals, Fig. 2 also shows fits based on analytical calculations ${ }^{23}$ of the amplitude and phase
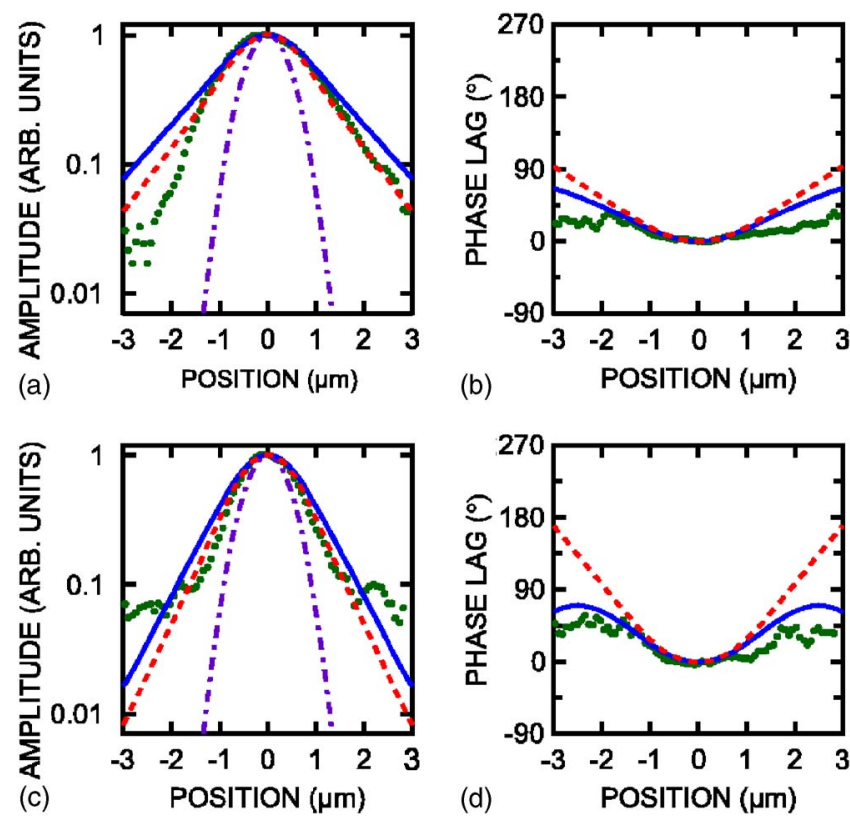

FIG. 2. (Color online) Measured amplitude and phase lag of the photothermally induced tip vibrations at $f_{1}-f_{2}=3 \mathrm{kHz}$, corresponding to a line scan (dots) across the spot center at $[(\mathrm{a})$ and (b)] chopping frequency $f_{1}=500 \mathrm{kHz}$ with $P=1 \mathrm{~mW}$ and $[(\mathrm{c})$ and (d) $] f_{1}=2 \mathrm{MHz}$ with $P=2 \mathrm{~mW}$. Also shown are the calculated amplitudes and phase lag of the surface displacement (solid lines) and the surface temperature (dashed lines) at $f_{1}$, and the optical intensity profile (dotted-dashed lines).

of the surface temperature (dashed lines) and the surface displacement (solid lines). The theory, based on the use of thermoelastic potentials and a quasistatic thermal expansion, assumes a Gaussian optical spot profile (dotted-dashed lines) and a sinusoidally varying heat absorption (at $f_{1}$ ) at the interface. The geometry is a single isotropic layer on a semiinfinite isotropic substrate, and we ignore any thermal boundary resistance $R_{\mathrm{th}}{ }^{24}$ First, the oscillating temperature field $T(x, y, z)$ is calculated, and then the surface displacement distribution $U(r)$ is obtained, where $r=\left(x^{2}+y^{2}\right)^{1 / 2}$ is the radius to the surface detection point. As an aside, for the simpler case of a semi-infinite solid, $U(r)$ is a sum over volume elements $d V^{\prime}$ at $\mathbf{r}^{\prime}=\left(x^{\prime}, y^{\prime}, z^{\prime}\right)$,

$$
U(r)=\frac{(1+\nu) \alpha}{\pi} \int T\left(\mathbf{r}^{\prime}\right) \frac{z^{\prime}}{\left|\mathbf{r}-\mathbf{r}^{\prime}\right|^{3}} d V^{\prime},
$$

where $\nu$ is the Poisson ratio and $\alpha$ is the linear thermal expansion coefficient. (Nowacki ${ }^{25}$ has derived a similar result for an infinite solid.) For our more complicated film-onsubstrate geometry, the formulas are not reducible to a compact form such as Eq. (1). We take literature values for $\alpha, \nu$, shear modulus, and thermal diffusivity $D_{s}$ of silica. ${ }^{26-28}$ Likewise for Cr (Refs. 26 and 27) except the thermal diffusivity $D_{f}$ because the thermal conductivities of thin films are known to differ strongly from bulk values. Using the data of Fig. 2 together with that of line scans at $100 \mathrm{kHz}$, $200 \mathrm{kHz}, 1 \mathrm{MHz}$, and $4 \mathrm{MHz}$ (not shown), we obtain $D_{f}=(4 \pm 2) \times 10^{-6} \mathrm{~m}^{2} \mathrm{~s}^{-1}$ by least-square fitting to the phase and to the logarithm of the amplitude over regions $(\sim \pm 1.5 \mu \mathrm{m})$ of reasonable signal-to-noise ratio. The optical spot size $W=1 \mu \mathrm{m}$ is taken directly from measurements with a knife edge. The derived value of $D_{f}$ for $\mathrm{Cr}$ is seven times smaller than the corresponding bulk value. ${ }^{26}$ This is not unexpected considering the effects of microstructure (inducing
to AlP license or copyright, see http://apl.aip.org/apl/copyright.jsp 

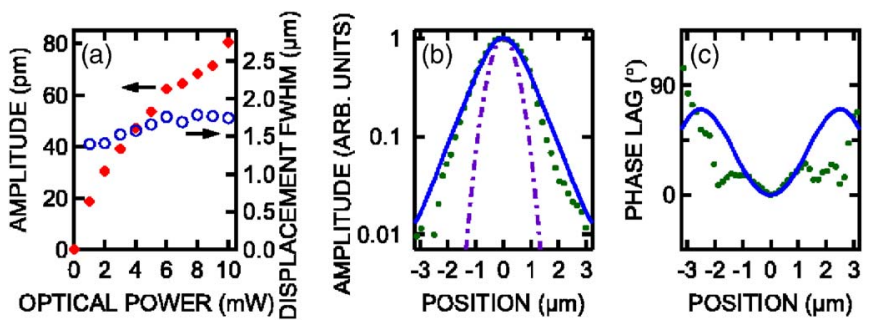

FIG. 3. (Color online) (a) Tip vibrational amplitude at $f_{1}-f_{2}=3 \mathrm{kHz}$ plotted (solid symbols) together with the FWHM of the amplitude-position curves (circles) vs incident optical power $P$ for $f_{1}=2 \mathrm{MHz}$. [(b) and (c)] Measured tip amplitude and phase lag profiles at $3 \mathrm{kHz}$ (dots) at $f_{1}=2 \mathrm{MHz}$ and $P$ $=8 \mathrm{~mW}$, and calculated amplitude and phase lag of the surface displacement (solid lines) at $f_{1}$ and spot profile (dotted-dashed lines).

grain-boundary scattering) or of contamination during sputtering (inducing impurity scattering) ${ }^{29}$ Moreover, estimates based on van der Pauw electrical resistivity measurements ${ }^{30}$ combined with the Wiedemann-Franz law ${ }^{31}$ gave comparably reduced values for the thermal conductivity. The quality of the fits in Fig. 2 based on the fitted value of $D_{f}$ is reasonable in the regions of significant signal.

The displacement amplitude varied linearly with the incident optical power up to $2 \mathrm{~mW}$, but showed signs of saturation for higher powers, as shown in Fig. 3(a) for $f_{1}=2 \mathrm{MHz}$. Interestingly, the FWHM width of the amplitude-position curves for displacement is not constant but increases with $P$ [see Fig. 3(a)]. Such an increase, which compensates the amplitude saturation so that the product of FWHM width and amplitude [the product of the two curves in Fig. 3(a)] is approximately proportional to the absorbed power, may be due to the temperature dependence of the sample or tip physical properties, or of the water meniscus between the tip and the sample. Examples of $Y$-directed scans obtained at the relatively high power $P=8 \mathrm{~mW}$ are shown in Figs. 3(b) and 3(c) for the amplitude and phase (dots), respectively. Because of the larger signal level, the signal-to-noise ratio is improved. The predicted amplitude and phase profiles for the displacement with the same value of $D_{f}$ as above are also shown for reference. The agreement is still reasonable in spite of the nonlinear behavior.

At present our apparatus is restricted in application to one-dimensional spatial profiling because of the fixed tip and moving mirror stage setup. However, by using a scanned tip and a fixed stage it should be possible to obtain twodimensional images of the thermoelastic field with nanoscale resolution. Since we are sensing surface displacement rather than temperature, the lateral resolution in the present setup is only limited by the mechanical contact area of nanometer order. Although it is difficult in arbitrary nanostructures to separate thermal diffusivity and thermal expansion, this method should prove useful for imaging nanoscale twodimensional megahertz thermal fields in thin films and in nanostructures of well-defined geometry.
The authors are grateful to Osamu Matsuda for helpful discussions and to The Mazda Foundation for support. Part of the work was done in the framework of an agreement between the Japanese Society for the Promotion of Science and the Consiglio Nazionale delle Ricerche of Italy for the support of the mobility of researchers.

${ }^{1}$ D. G. Cahill, H. E. Fischer, T. Klitsner, E. T. Swartz, and R. O. Pohl, J. Vac. Sci. Technol. A 7, 1259 (1989).

${ }^{2}$ J. C. Lambropoulos, M. R. Jolly, C. A. Amsden, S. E. Gilman, M. J. Sinicropi, D. Diakomihalis, and S. D. Jacobs, J. Appl. Phys. 66, 4230 (1989).

${ }^{3}$ K. E. Goodson and M. I. Flik, Appl. Mech. Rev. 47, 101 (1994).

${ }^{4}$ J. Opsal, A. Rosencwaig, and D. L. Willenborg, Appl. Opt. 22, 3169 (1983).

${ }^{5}$ C. A. Paddock and G. L. Eesley, J. Appl. Phys. 60, 285 (1986).

${ }^{6}$ E. P. Visser, E. H. Versteegen, and W. J. P. van Enckevort, J. Appl. Phys. 71, 3238 (1992).

${ }^{7}$ O. W. Kading, H. Skurk, A. A. Maznev, and E. Matthias, Appl. Phys. A: Mater. Sci. Process. 61, 253 (1995).

${ }^{8}$ S. Dilhaire, D. Fournier, and G. Tessier, in Microscale and Nanoscale Heat Transfer, Topics in Applied Physics Vol. 107, edited by S. Volz (Springer, Berlin, 2007), p. 239.

${ }^{9}$ B. Li, L. Pottier, J. P. Roger, D. Fournier, and E. Welsch, Rev. Sci. Instrum. 71, 2154 (2000).

${ }^{10}$ W. B. Jackson, N. M. Amer, A. C. Boccara, and D. Fournier, Appl. Opt. 20, 1333 (1981).

${ }^{11}$ E. Welsch and M. Reichling, J. Mod. Opt. 40, 1455 (1993).

${ }^{12}$ Q. Shen, A. Harata, and T. Sawada, Jpn. J. Appl. Phys., Part 1 35, 2339 (1996).

${ }^{13}$ J. Jumel, F. Taillade, and F. Lepoutre, Eur. Phys. J.: Appl. Phys. 23, 217 (2003).

${ }^{14}$ A. Majumdar, Annu. Rev. Mater. Sci. 29, 505 (1999).

${ }^{15}$ H. M. Pollock and A. Hammiche, J. Phys. D 34, R23 (2001).

${ }^{16}$ O. Kwon, L. Shi, and A. Majumdar, J. Heat Transfer 125, 156 (2003).

${ }^{17}$ S. Lefèvre and S. Volz, Rev. Sci. Instrum. 76, 033701 (2005).

${ }^{18}$ J. Bolte, F. Niebisch, J. Pelzl, P. Stelmaszyk, and A. D. Wieck, J. Appl. Phys. 84, 6917 (1998).

${ }^{19}$ M. Tomoda, N. Shiraishi, O. V. Kolosov, and O. B. Wright, Appl. Phys. Lett. 82, 622 (2003).

${ }^{20}$ O. B. Wright and K. Kawashima, Phys. Rev. Lett. 69, 1668 (1992).

${ }^{21}$ We neglect the defocusing caused by scanner motion that gives rise to an estimated maximum $2 \%$ increase in $W$.

${ }^{22} \mathrm{We}$ use subpicosecond optical pulses with $82 \mathrm{MHz}$ repetition rate, but this has no influence on the sub-10 MHz dynamics here.

${ }^{23} \mathrm{R}$. Li Voti, O. B. Wright, and M. Tomoda (unpublished).

${ }^{24}$ Simulations showed that owing to the low substrate thermal diffusivity, the incorporation of any value of $R_{\mathrm{th}}$, even infinite, changed the FWHM of the temperature profiles by $<10 \%$ for a broad range of $\mathrm{Cr}$ diffusivities.

${ }^{25} \mathrm{~W}$. Nowacki, Thermoelasticity (Pergamon, London, 1962), pp. 10-13.

${ }^{26}$ American Institute of Physics Handbook, 3rd ed., edited by D. E. Gray (McGraw-Hill, New York, 1972), pp. 3-107, 4-108, 4-135, 4-154, and 4-158.

${ }^{27}$ G. S. Kino, Acoustic Waves: Devices, Imaging and Analog Signal Processing (Prentice-Hall, New Jersey, 1987), p. 549.

${ }^{28}$ O. B. Wright, R. Li Voti, O. Matsuda, M. C. Larciprete, C. Sibilia, and M. Bertolotti, J. Appl. Phys. 91, 5002 (2002).

${ }^{29}$ A. K. Kulkarni and L. C. Chang, Thin Solid Films 301, 17 (1997).

${ }^{30}$ L. J. van der Pauw, Philips Res. Rep. 13, 1 (1958).

${ }^{31}$ C. Kittel, Introduction to Solid State Physics, 7th ed. (Wiley, New York, 1996), pp. 166-168. 